\title{
Laser ablated coupling structures for optical printed circuit boards
}

\author{
Geert Van Steenberge ${ }^{\mathrm{a}}$, Peter Geerinck ${ }^{\mathrm{a}}$, Markus Riester ${ }^{\mathrm{b} *}$, Siegfried Pongratz ${ }^{\mathrm{b}}$, Peter Van Daele ${ }^{\mathrm{a}}$ \\ ${ }^{\mathrm{a}}$ Ghent University, TFCG Microsystems, Dept. of Information Technology, B-9052 Ghent, Belgium \\ ${ }^{b}$ Motorola GmbH, Motorola Labs - PRRC-Europe, D-65232 Taunusstein, Germany
}

\begin{abstract}
We report on the cost effective fabrication of $45^{\circ}$ micromirror couplers within single-mode polymer waveguides for achieving fully embedded board-level optoelectronic interconnections. Compatibility with existing board manufacturing technology is achieved by making use of polymers with high thermal stability. The sol-gel polymers behave as negative photo resist and waveguides are patterned by UV exposure. Micromirrors are fabricated using excimer laser ablation, a very flexible technology that is particularly well suited for structuring of polymers because of their excellent UVabsorption properties and highly non-thermal ablation behavior. A coupling structure based on total internal reflection (TIR) is enhanced by developing a process for embedding a metal coated $45^{\circ}$ mirror in the optical layers. The mirrors are selectively metallized using a lift-off process. Filling up the angled via without the presence of air bubbles and providing a flat surface above the mirror is only possible by enhancing the cladding deposition process with ultrasound agitation. Surface roughness of both the mirrors and the upper cladding surface above the mirrors is investigated using a non-contact optical profiler. Initial loss measurements at $1.3 \mu \mathrm{m}$ show a propagation loss of $0.62 \mathrm{~dB} / \mathrm{cm}$ and an excess mirror loss of $1.55 \mathrm{~dB}$. During most recent experiments mirror roughness has been reduced from $160 \mathrm{~nm}$ to $20 \mathrm{~nm}$, which will seriously reduce the mirror loss.
\end{abstract}

Keywords: laser ablation, optical interconnect, polymer waveguide, printed circuit board (PCB), $45^{\circ}$ mirror

\section{INTRODUCTION}

Optical data transmission has become a mainstream technology for broadband core and metro networks. While the technology is still expensive, it is becoming more and more attractive for systems close to the customer by introducing all-optical technologies. Using standard production processes, but using materials with enhanced compatibility, and using novel system and device designs will make optoelectronic devices and systems more affordable, and thus will be driving their deployment also to access networks. The target of our research is to develop PCB integrated optical devices that drive the implementation of optical network equipment in the last mile of cable TV CATV and Internet Protocol (IP) access networks at competitive costs. This refers to cost across the whole life cycle of the product: materials, manufacturing, assembly, installation, operation and end-of-life. The research will evaluate existing and novel optical materials and take advantage of industry standard high-density integration and explore new technologies.

While many of the objectives of optical data connections on PCBs have been addressed at length by many groups [1-8], the cost effective manufacturing of coupling structures for guiding light into optical waveguides has still not been solved in a way that would enable optical PCBs reach a state where broad implementation can be expected. Different solutions provide highly parallel optical chip-to-chip data transmission [7]; other solutions provide interconnections based on MT ferrule design [9]. The most critical point that needs to be addressed is the low-cost manufacturing of the interconnection and the compatibility with current PCB production. The latter refers to the processes as well as material compatibility.

The redirection of light takes a central role in the optical interconnect solution. Many solutions can be found in the scientific and patent literature for guiding the light out of the plane. These include embedded fibers bent to redirect light perpendicular to the surface. Other solutions favor the "periscope" $[1,3]$ where chips carry the active devices and guide light directly into and out of the embedded optical waveguide, and many others. Our investigation concentrates on the solution where a $45^{\circ}$ mirror is used for redirecting the light, and the particular manufacturing method that allows easy integration into the PCB manufacturing process.

* Current address: AT\&S AG, Fabriksgasse 13, A-8700 Leoben, Austria

Integrated Optics: Theory and Applications, edited by Tadeusz Pustelny, Paul V. Lambeck, Christophe Gorecki, Proc. of SPIE Vol. 5956, 59561A, (2005) · 0277-786X/05/\$15 - doi: 10.1117/12.622890 


\section{SOL-GEL POLYMER WAVEGUIDE TECHNOLOGY}

Polymer materials are generally recognized to exhibit various favorable properties for the use in optical waveguide technology. There is a great potential in terms of optical properties, cost-effectiveness, and processing possibilities. Different classes of eligible polymers are acrylates, siloxanes, polyimides, polycarbonates, and olefins. With regard to optical board applications polymers have to fulfill tight requirements, such as very low optical losses and the compatibility with PCB manufacturing processes (e.g. solder reflow and lamination process).

Allowing for these low losses and the required compatibility with PCB manufacturing, we have chosen the use of hybrid inorganic-organic materials $\left(\right.$ Ormocer $^{\circledR}$ ) [10], commercially available. They consist of inorganic networks (silica) additionally cross-linked by organic side chains. The materials are easy tunable in the refractive index and cross-linked by UV-exposure after spin coating. The most important properties of the Ormocer ${ }^{\circledR}$-materials to be used for board-level optical interconnects are listed in TABLE I. Material losses are $0.3 \mathrm{~dB} / \mathrm{cm}$ at $1320 \mathrm{~nm}$, and $0.6 \mathrm{~dB} / \mathrm{cm}$ at $1550 \mathrm{~nm}$.

TABLE I

\begin{tabular}{ll}
\hline Attenuation & $0.3 \mathrm{~dB} / \mathrm{cm}$ at $1320 \mathrm{~nm}$ \\
& $0.6 \mathrm{~dB} / \mathrm{cm}$ at $1550 \mathrm{~nm}$ \\
Decomposition temperature & $270{ }^{\circ} \mathrm{C}$ \\
Core index tuning range & 1.519 to 1.537 at $1320 \mathrm{~nm}, 1550 \mathrm{~nm}$ \\
Water absorption & $<0.5 \%$ \\
CTE $\left(20-100^{\circ} \mathrm{C}\right)$ & $100-130 \mathrm{ppm} / \mathrm{K}$ \\
Shrinkage during curing & $2-8 \mathrm{Vol} \%$ \\
\hline
\end{tabular}

Selected properties of Ormocer ${ }^{\circledR}$-materials to be used for board-level optical interconnects

Single mode optical waveguide fabrication was done by the Fraunhofer Institute for Applied Optics and Precision Engineering (IOF), Germany. Only a very short overview of the processing steps is given. The deposition of the liquid polymeric layers as claddings and waveguide cores on standard halogen-free FR4 PCB substrates is done by spincoating. The patterning of the core layer is performed photographically by UV exposure through a mask in proximity and by subsequent solvent developing. Single mode waveguides with cross-sectional areas of $7 \mathrm{x} 8 \mu \mathrm{m}^{2}$ are used as testbed structures for the vertical coupling structures. A cross-section is given in Fig.1.

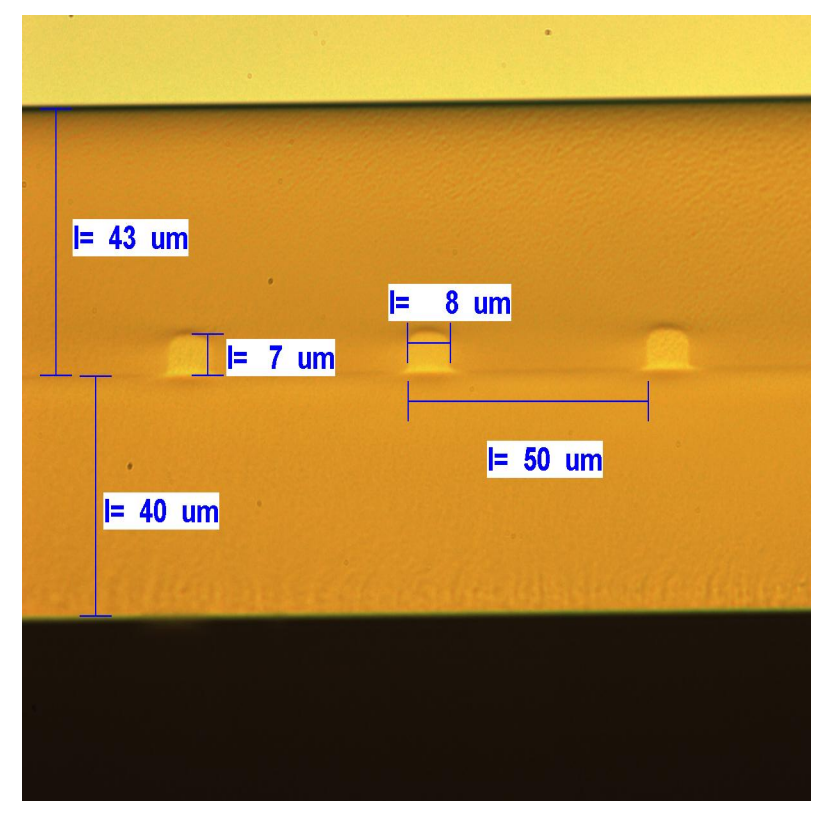

Fig.1. Embedded waveguides on silicon substrate, allowing cross-sections without polishing steps 


\section{COUPLING STRUCTURES}

The manufacturing of coupling structures for guiding light into the optical waveguides has still not been solved in a way that would enable optical PCBs reach a state where broad implementation can be expected. The most critical point that needs to be addressed is the low-cost manufacturing and the compatibility with current PCB production. The many methods used for redirecting light into the PCB up to this point [1-3] failed to provide a cost-effective solution to the interconnect problem. Our investigation therefore concentrates on the solution where a $45^{\circ}$ mirror is used for redirecting the light, and the particular manufacturing method that allows easy integration into the PCB manufacturing process.

There are various techniques to fabricate $45^{\circ}$ micromirrors. Micro machining techniques using a $90^{\circ} \mathrm{V}$-shaped diamond blade can provide an acceptable cut surface, but due to the physical size of the machining tool, it is difficult to cut individual waveguides on the same substrate. Reactive ion etching (RIE) techniques where the slope of the mirror is formed by $45^{\circ}$ oblique etching is limited by directional freedom. Temperature controlled RIE is not limited by directional freedom but this method has the disadvantage of being material dependent. Dry etching techniques are not favored because of the non-compatibility of the process with standard PCB manufacturing processes. The technique we propose for the fabrication of the optical coupling structures is the use of laser ablation, already being used in PCB manufacturing for the drilling of microvia's in high density boards and trimming of embedded resistors. This means that the technology is fully compatible with standard electrical PCB manufacturing which is an important advantage compared to other technologies.

Laser ablation is a micromachining technology that is based on the controlled removal of material with intense laser pulses. In general, the energy of the laser beam is absorbed in a thin layer or small volume and the ablation process takes place through rapid fragmentation. Depending on the wavelength and the material, this can have the characteristics of ablative photo-decomposition, or rapid heating and vaporization. Our unique ablation set-up contains three different laser sources: a $\mathrm{KrF}$ excimer-, a frequency tripled $\mathrm{Nd}$ :YAG- and a $\mathrm{CO}_{2}$-laser, being able to structure a whole set of materials in different applications. KrF excimer laser ablation (wavelength $248 \mathrm{~nm}$ ) is particularly well suited for structuring of polymers because of their excellent UV-absorption properties and highly non-thermal ablation behavior. In our setup, simply changing the angle of a turning mirror can tilt the excimer laser beam. Each facet is fabricated by sending the laser beam through a rectangular aperture that is projected on the sample.

Two basic coupling structures are proposed in Fig.2. The first coupling structure is based on total internal reflection (TIR) at the interface between the Ormocer ${ }^{\mathbb{B}}$-material and the air gap (negative facet), in the second coupling structure the light beam is reflected on a metal coated mirror (positive facet), embedded in the PCB.

An SEM picture of a micromirror based on total internal reflection is shown in Fig.3. It can be seen that using the excimer laser, there is always a certain tapering of the edges. The tapering effect is shown to be highly reproducible, therefore this effect can be compensated for in order to achieve an angle of $45^{\circ}$ at the negative facet. TABLE II gives an overview of the ablation parameters. Fabrication time of one mirror is about $1 \mathrm{sec}$.

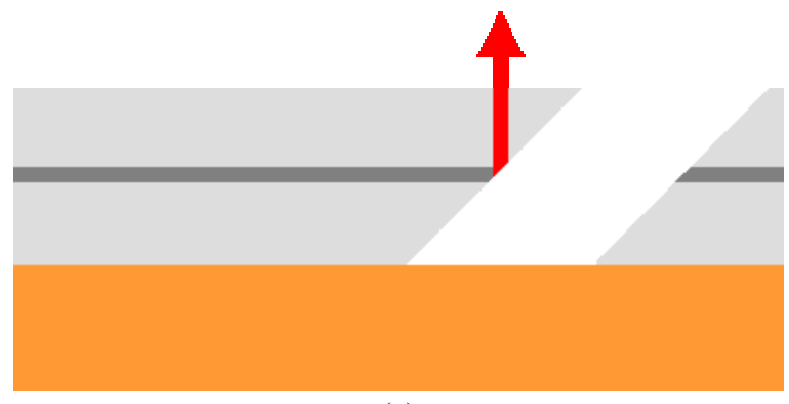

(a)

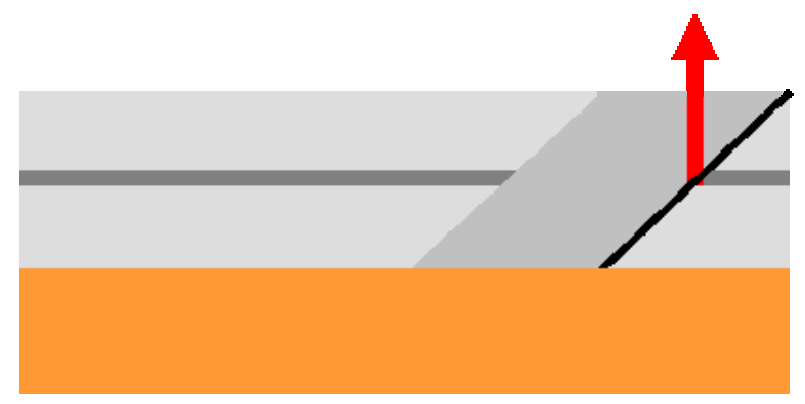

(b)

Fig.2. Schematic view of two coupling concepts (a) mirror based on total internal reflection (negative facet), (b) metal coated mirror embedded in the PCB (positive facet) 


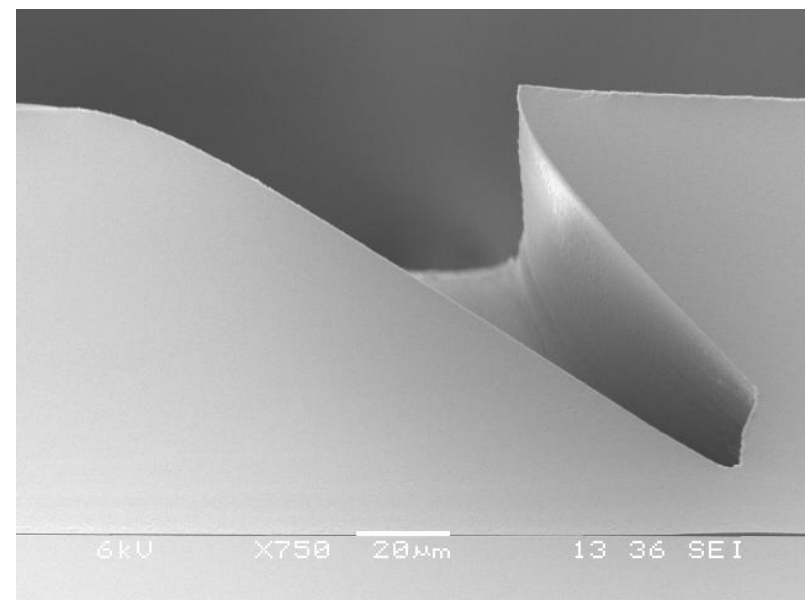

Fig.3. SEM picture of a cross-section of a micromirror fabricated in Ormocer $^{\circledR}$ using a $\mathrm{KrF}$ excimer laser

\begin{tabular}{ll}
\multicolumn{2}{c}{ TABLE II } \\
\hline Fluence & $2.5 \pm 0.5 \mathrm{~J} / \mathrm{cm}^{2}$ \\
Repetition Rate & $200 \mathrm{~Hz}$ \\
Number of pulses & 290 \\
Tapering compensation & $9^{\circ}$ \\
\hline
\end{tabular}

Parameters for $\mathrm{KrF}$ excimer laser ablation of a TIR $45^{\circ}$ micromirror in Ormocer ${ }^{\circledR}$

Fig. 4 shows a WYKO plot (non-contact optical profiler, available in the Department of Applied Physics and Photonics, Vrije Universiteit Brussel, Belgium) of a laser-ablated micromirror (positive facet). The Ra roughness of the mirror on an area of $50 \times 50 \mu \mathrm{m}^{2}$ is $80 \mathrm{~nm}$ and the RMS roughness $110 \mathrm{~nm}$, with good reproducibility of $\pm 10 \mathrm{~nm}$.

The condition for TIR of the light beam at the negative facet is very critical. Light will not be reflected when the condition is not reached. This can be the case if an adhesive penetrates into the hole during a later mounting phase. Humidity could also be a problem. For these reasons it might be necessary to apply a metal reflection coating. The overview of the processing steps for the fabrication of a coated mirror embedded in the optical layers is given in Fig.5. Selective coating of only the positive facet is possible using a lift-off process. A photo-resist layer is applied prior to ablation of the angled optical via. Evaporation of $500 \mathrm{~nm}$ gold is used as coating process. Due to the directional selectivity of the evaporation process, the negative facet is not coated. After development of the resist a coated positive facet can be used as deflecting mirror.
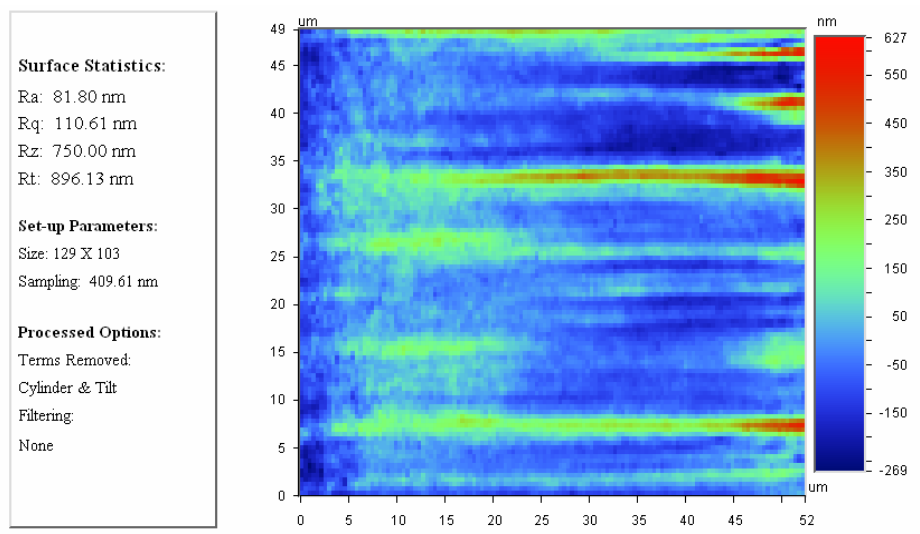

Fig.4. WYKO plot to analyze surface roughness of a micromirror fabricated in Ormocer ${ }^{\circledR}$ using a KrF excimer laser 


\section{Fabrication optical waveguiding structure on PCB}

\begin{tabular}{|c|}
\hline Application photo-resist \\
Excimer laser ablation angled optical via \\
Gold evaporation \\
Development resist \\
\hline
\end{tabular}

\begin{tabular}{|c|}
\hline Fill-up of cavity with cladding material \\
Ultrasound agitation \\
Spin-coating \\
Curing \\
\hline
\end{tabular}
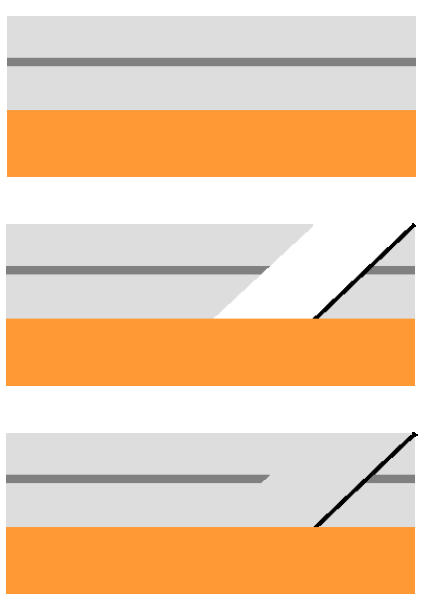

Fig.5. Technology sequence for the fabrication of a metal coated mirror embedded in the optical layer

For a reliable functioning it was assumed that the presence of air bubbles in the cavity could be detrimental due to the temperature dependence of the pressure within the bubbles. Therefore, ways of filling up the cavity without the presence of air bubbles were studied. At the same time, providing a flat surface above the mirror is another important feature required for subsequent processes and has to be regarded as well. It was found that filling the cavity without air bubbles was only possible by enhancing the cladding deposition process with ultrasound agitation, because of the higher viscosity of the Ormocer ${ }^{\circledR}$-material. During the investigation it could be shown that even small air bubbles will cause reliability problems. The final curing step of the additional cladding material at $170^{\circ} \mathrm{C}$ will lead to a volume expansion of the air bubble, resulting in a surface elevation (Fig.6a). Ultrasound agitation is needed for at least 8 minutes to avoid the presence of air bubbles at the bottom of the cavity (Fig.6b). Afterwards, a spin-coating step is necessary to achieve a flat surface. In Fig.6b spin coating speed was $8000 \mathrm{rpm}$, resulting in an additional layer thickness of $2 \mu \mathrm{m}$. Lower spinning speeds (4000 rpm) show optimum results, as the additional cladding layer thickness is about $5 \mu \mathrm{m}$, which is enough to resist against mechanical stresses present during handling.

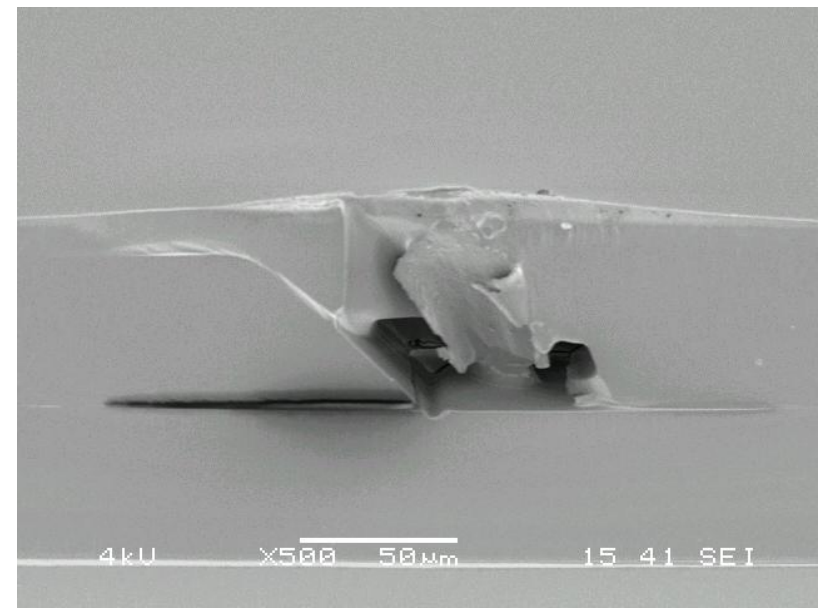

(a)

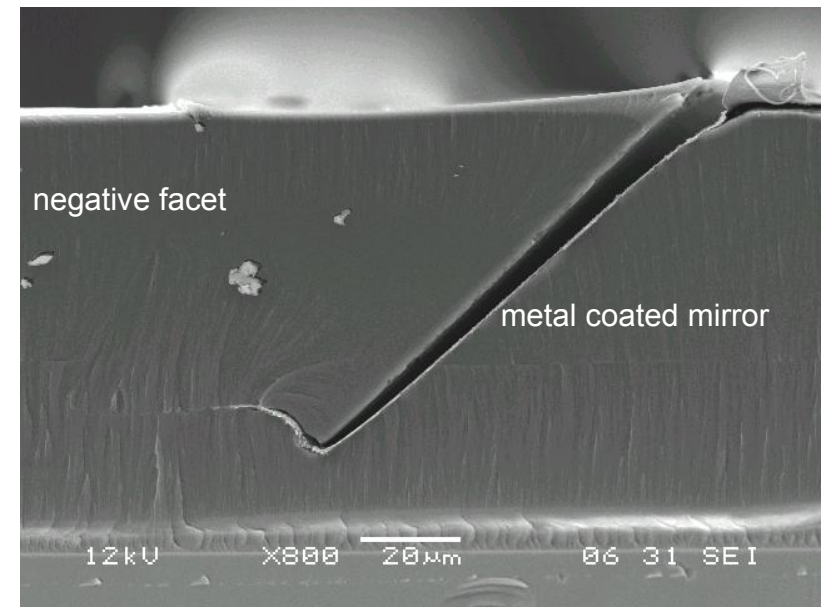

(b)

Fig.6. Effect of ultrasound agitation and spin-coating on filling up the angled optical via with Ormocer ${ }^{\circledR}$-cladding material (a) no ultrasound agitation results in the presence of air in the cavity leading to poor system performance and to surface elevation at higher temperatures (b) result of 8 minutes of ultrasound agitation and spin-coating at $8000 \mathrm{rpm}$, mechanical stresses introduced during cleaving the sample (to make the cross-section) caused the crack in between the gold mirror and the additional cladding material, this is not the case in real life situations 

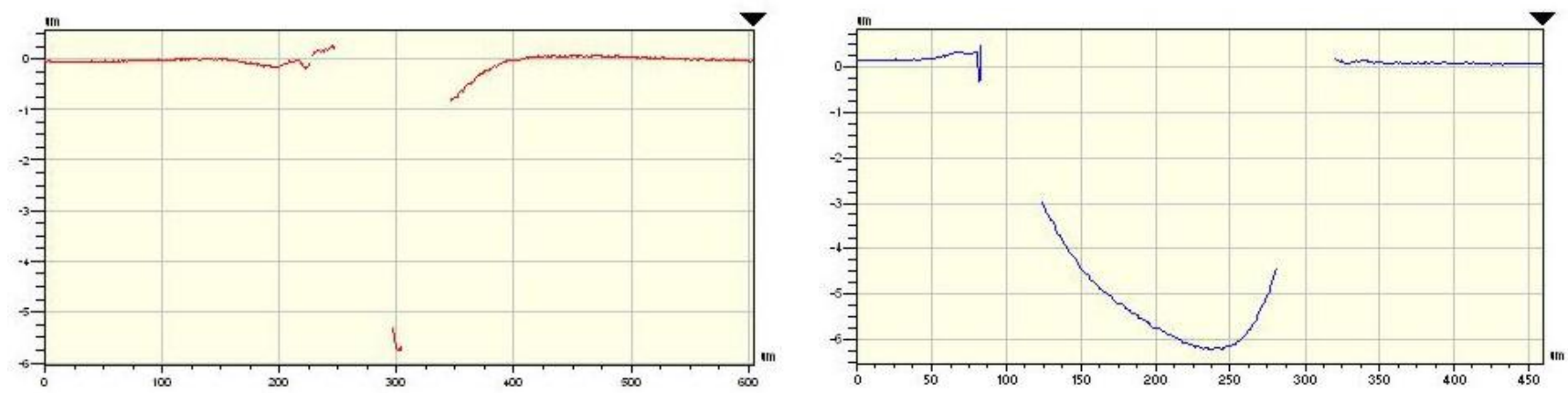

Fig.7. WYKO plot to analyze surface flatness above an embedded metal coated mirror

The flatness of the upper cladding surface above the embedded mirror has been investigated using a non-contact optical profiler. The result is shown in Fig.7. A height difference of $6 \mu \mathrm{m}$ on a distance of $200 \mu \mathrm{m}$ (X direction) or $400 \mu \mathrm{m}$ (Y direction) can be observed, which should be sufficient to provide a mounting surface for any opto-electronic component.

Ablation of a positive facet has slightly different parameters compared to ablation of a negative facet. Due to the tapering, the actual ablation angle is $34^{\circ}$; the number of pulses is 270 . The accuracy of the $45^{\circ}$ mirror surface is $\pm 1^{\circ}$, which should be sufficient, both for total internal reflection, and for a metal coated mirror.

Surface roughness of the mirror after coating is slightly higher compared to non-coated mirrors, most probably introduced during the additional processing steps: gold evaporation and development of photo resist. The RMS roughness of a coated mirror on an area of $50 \times 50 \mu \mathrm{m}^{2}$ is $160 \mathrm{~nm} \pm 20 \mathrm{~nm}$, based on 3 measurements.

\section{MEASUREMENTS}

Both the propagation loss and the excess loss of a vertical out-coupling facet compared to a polished horizontal outcoupling facet are determined, at $1.3 \mu \mathrm{m}$. A cleaved single mode fiber is placed in front of a polished horizontal incoupling facet. No index matching adhesives are used to eliminate Fresnel losses. At the out-coupling facet, the detector is placed in front of the facet, both for horizontal and vertical out-coupling. The cut-back method is used to determine the attenuation of the single mode waveguides integrated on the PCB, at a length of $3.2 \mathrm{~cm}, 2 \mathrm{~cm}$ and $1.4 \mathrm{~cm}$, showing an average attenuation loss of $0.62 \mathrm{~dB} / \mathrm{cm}$ and a standard deviation of $0.08 \mathrm{~dB} / \mathrm{cm}$, as shown in Fig.8. The higher attenuation loss compared to the specifications (see TABLE I, $0.3 \mathrm{~dB} / \mathrm{cm}$ at $1.3 \mu \mathrm{m}$ ) might be due to the higher surface roughness of the PCB substrate compared to that of a silicon substrate, but this still needs to be verified. Coupling loss for two polished facets is $2.76 \mathrm{~dB}$. Coupling loss could be further reduced by matching the waveguide mode diameter to that of the single mode fiber, and by using index matching adhesives.

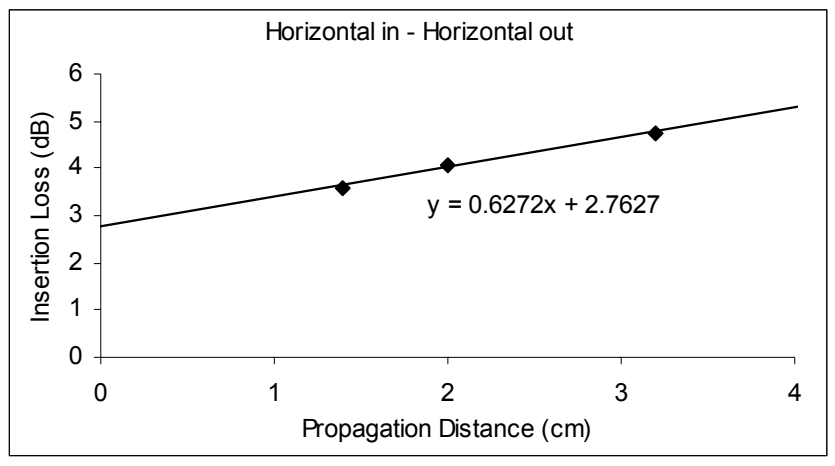

Fig.8. Propagation loss at $1.3 \mu \mathrm{m}$ of single mode polymer waveguides integrated on a PCB, measured using the cut-back principle (horizontal in- and out-coupling) 
The excess loss of a metal coated vertical out-coupling mirror compared to a polished horizontal out-coupling facet is $1.55 \mathrm{~dB}$, based on most consistent measurements out of initial results. This excess loss is quite high, but surface roughness measurements have shown the relatively high roughness of a coated mirror $(160 \mathrm{~nm} \pm 20 \mathrm{~nm})$. Latest experiments show a surface roughness of $20 \mathrm{~nm}$ on an area of $40 \times 60 \mu \mathrm{m}^{2}$, which will seriously reduce the excess loss of the vertical out-coupling mirrors. This will be further investigated in future work.

\section{CONCLUSION}

A process for embedding metal coated $45^{\circ}$ micromirrors in optical waveguiding layers has been developed. Low-cost manufacturing and compatibility with current PCB production is achieved. Angled optical vias are fabricated using excimer laser ablation. Mirrors are selectively metallized using a lift-off process. Filling up the angled via without the presence of air bubbles and providing a flat surface above the mirror is only possible by enhancing the cladding deposition process with ultrasound agitation. Surface roughness of both the mirrors and the upper cladding surface above the mirrors is investigated using a non-contact optical profiler. Initial loss measurements at $1.3 \mu \mathrm{m}$ show a propagation loss of $0.62 \mathrm{~dB} / \mathrm{cm}$ and an excess mirror loss of $1.55 \mathrm{~dB}$. During most recent experiments mirror roughness has been reduced from $160 \mathrm{~nm}$ to $20 \mathrm{~nm}$, which will seriously reduce the mirror loss.

\section{ACKNOWLEDGEMENT}

This work has been partially supported by the Motorola Physical Realization Research Center - Europe, and partially by the IWT (Institute for the Promotion of Innovation by Science and Technology in Flanders), in the framework of the project "Plastic for Photonics". The authors would like to thank the Fraunhofer Institute for Applied Optics and Precision Engineering (IOF) for supplying single mode waveguide samples; the Department of Applied Physics and Photonics (TW-TONA), Vrije Universiteit Brussel, for the use of their non-contact optical profilometer; the Photonics research group, Department of Information Technology, Ghent University, for the use of their measurement equipment.

\section{REFERENCES}

1. E. Griese, A High-Performance Hybrid Electrical-Optical Interconnection Technology for High-Speed Electronic Systems, IEEE Transactions on Advanced Packaging, Vol. 24, No. 3, pp. 375-383, 2001.

2. F. Mederer, R. Jager, H. J. Unold, R. Michalzik, K. J. Ebeling, S. Lehmacher, A. Neyer, and E. Griese, 3-Gb/s data transmission with GaAs VCSEL's over PCB integrated polymer waveguides, IEEE Photonics Technology Letters, Vol. 13, No. 9, pp. 1032-1034, 2001.

3. D. Krabe, F. Ebling, N. Arndt-Staufenbiel, G. Lang, W. Scheel, New Technology for Electrical/Optical Systems on Module and Board Level: The EOCB Approach, Proc. ECTC 2000, pp.970-974.

4. G.L. Bona, B.J. Offrein, U. Bapst, C. Berger, R. Beyeler, R. Budd, R. Dangel, L. Dellmann, and F. Horst, Characterization of parallel optical-interconnect waveguides integrated on a printed circuit board, Proc. of SPIE, Vol. 5453, pp. 134-141, 2004.

5. B. Lunitz, J. Guttmann, H.P. Huber, J. Moisel, M. Rode, Experimental Demonstration of $2.5 \mathrm{Gbit} / \mathrm{s}$ Transmission with $1 m$ Polymer Optical Backplane, Electronics Letters, Vol. 37, No. 17, pp. 1079, 2001

6. For more information on the Network of Excellence on Micro-Optics, see http://www.micro-optics.org

7. For more information on the IO project, see http://io.intec.ugent.be

8. P. Lukowicz, J. Jahns, R. Barbieri, P. Benabes, T. Bierhoff, A. Gauthier, M. Jarczynski, G.A. Russell, J. Schrage, W. Süllau, J.F. Snowdon, M. Wirz, and G. Tröster, Optoelectronic Interconnection Technology in the HOLMS System, IEEE Journal on Selected Topics in Quantum Electronics, Vol. 9, No. 2, pp.624-635, 2003.

9. G. Van Steenberge, P. Geerinck, S. Van Put, J. Van Koetsem, H. Ottevaere, D. Morlion, H. Thienpont, P. Van Daele, MT-Compatible Laser-Ablated Interconnections for Optical Printed Circuit Boards, Journal of Lightwave Technology, Vol. 22, No. 9, pp. 2083-2090, 2004.

10. R. Buestrich, F. Kahlenberg, M. Popall, P. Dannberg, R. Muller-Fiedler, O. Rosch, ORMOCER (R) s for optical interconnection technology, Journal of Sol-Gel Science and Technology, Vol. 20, No. 2, pp. 181-186, 2001. 